\title{
AXIOMATIC APPROACH TO THE HOMOTOPY GROUPS
}

\section{SZE-TSEN HU}

1. Introduction. The history of homotopy groups traces back to the fundamental groups of Poincaré $[9 ; 10 ; 14] .{ }^{1}$ For a given topological space $X$ and a given point $x_{0}$ in $X$, the funnamental group $\pi_{1}\left(X, x_{0}\right)$ is defined by considering the loops in $X$ with $x_{0}$ as basic point, i.e., the continuous maps $\lambda: S^{1} \rightarrow X$ of the circle $S^{1}$ into $X$ with a given point $s_{0}$ of $S^{1}$ mapped into $x_{0}$.

Replacing the circle $S^{1}$ by a higher dimensional sphere $S^{n}$, Hurewicz [7] introduced the homotopy groups $\pi_{n}\left(X, x_{0}\right)$ in 1935 which turned out to be very useful and prolific. In 1941, relative homotopy groups $\pi_{n}\left(X, A, x_{0}\right), n \geqq 2$, of a topological space $X$ modulo a subspace $A$ at a given point $x_{0}$ were introduced by a joint paper of Hurewicz and Steenrod [4] and also independently by J. H. C. Whitehead [16]. These groups are defined by considering the continuous maps of an $n$-dimensional cell $E^{n}$ into $X$ with the boundary sphere $S^{n-1}$ mapped into $A$ and a given point $s_{0}$ of $S^{n-1}$ mapped into $x_{0}$.

For each $n \geqq 2$, a boundary homomorphism

$$
\partial: \pi_{n}\left(X, A, x_{0}\right) \rightarrow \pi_{n-1}\left(A, x_{0}\right)
$$

is defined by taking restrictions of the maps on the boundary sphere $S^{n-1}$ of the cell $E^{n}$.

For any continuous map $f:\left(X, A, x_{0}\right) \rightarrow\left(Y, B, y_{0}\right)$, an induced homomorphism

$$
f_{*}: \pi_{n}\left(X, A, x_{0}\right) \rightarrow \pi_{n}\left(Y, B, y_{0}\right)
$$

is defined by means of composition.

These are the entities of the so-called homotopy theory [5]. One observes that the homotopy theory looks quite like a homology theory.

Since Eilenberg and Steenrod [2] established their celebrated axiomatic approach to homology theory in 1945 , it has been a natural problem to ask whether a similar approach is possible for homotopy theory.

An address delivered before the Knoxville meeting of the Society on November 18, 1955, by invitation of the Committee to Select Hour Speakers for Southeastern Sectional Meetings; received by the editors February 23, 1956.

${ }^{1}$ Numbers in brackets refer to the bibliography at the end of the paper. 
Because of the obvious similarity between homotopy theory and homology theory, one would naturally first ask whether the axioms of Eilenberg and Steenrod hold in homotopy theory. A check of the axioms shows that all but one of the axioms are satisfied. The exception is the excision axiom. Simple examples can be constructed where the excision axiom is violated. See [13, p. 83].

Steenrod, in his book [13], suggested that a candidate to replace the excision axiom might be the fibering theorem in the theory of fiber bundles $[13$, p. 90, Theorem 17.1]. His conjecture is almost completely correct except that the notion of fiber bundles is too strong to include the spaces needed in the various constructions contained in the uniqueness proof unless the category of spaces is restricted to consist of all CW-complexes only.

If, in Steenrod's conjecture, one replaces fiber bundles by fiber spaces in the generalized sense of Serre [11, p. 443], then a complete axiomatic approach may be established. In the present address, such an axiomatic approach will be sketched. ${ }^{2}$ In fact, only a very special kind of fiber space will be used, namely, the space of paths.

2. Preliminaries. By a triplet $\left(X, A, x_{0}\right)$, we mean a topological space $X$, a nonvacuous subspace $A$ of $X$, and a point $x_{0}$ in $A$. If $A=x_{0}$, then the triplet $\left(X, A, x_{0}\right)$ will be simply denoted by $\left(X, x_{0}\right)$ and may be considered as a pair consisting of a topological space $X$ and a point $x_{0}$ in $X$.

Let $\left(X, A, x_{0}\right)$ be a given triplet. Consider the set $P(X)$ of all pathcomponents of $X$. The path-components of $X$ containing points of $A$ form a subset $O$ of $P(X)$. If we identify $O$ to be a single point, we obtain a quotient set $P(X, A)$ which will be called the set of pathcomponents of $X$ modulo $A$. If $A=x_{0}$, then $P(X, A)=P(X)$.

Next, let $U$ denote the space of all paths in $X$ issuing from $x_{0}$; that is to say, $U$ consists of the continuous maps $\sigma: I \rightarrow X$ with $\sigma(0)=x_{0}$, where $I=[0,1]$ denotes the closed unit interval, and is topologized by the compact-open topology. There is a natural map $p: U \rightarrow X$ defined by $p(\sigma)=\sigma(1)$. Let $C=p^{-1}(A)$ and $u_{0}$ denote the degenerate path $u_{0}(I)=x_{0}$. Thus we obtain a triplet $\left(U, C, u_{0}\right)$ called the associated triplet of $\left(X, A, x_{0}\right)$. The map $p$ defines a continuous map

$$
p:\left(U, C, u_{0}\right) \rightarrow\left(X, A, x_{0}\right)
$$

called the associated projection.

2 The essential idea of this axiomatic approach was given independently by J.-P. Serre and J. Milnor. See [17]. 
Let $\left(Y, B, y_{0}\right)$ be a second triplet and let $f:\left(X, A, x_{0}\right) \rightarrow\left(Y, B, y_{0}\right)$ be a given continuous map. Since $f$ maps the path-components of $X$ into those of $Y$, it induces a natural induced transformation

$$
f_{*}: P(X, A) \rightarrow P(Y, B)
$$

in an obvious way. $f_{*}$ maps the neutral element of $P(X, A)$ into that of $P(Y, B)$.

Let $\left(V, D, v_{0}\right)$ denote the associated triplet of $\left(Y, B, y_{0}\right)$ with the associated projection

$$
r:\left(V, D, v_{0}\right) \rightarrow\left(Y, B, y_{0}\right) .
$$

Then $f$ induces a continuous map $\phi:\left(U, C, u_{0}\right) \rightarrow\left(V, D, v_{0}\right)$ defined by $\phi(\sigma)=f \sigma$ for each $\sigma$ in $U$. Obviously, $f p=\phi r$ holds in the following rectangle

$$
\begin{gathered}
\left(U, C, u_{0}\right) \stackrel{\phi}{\rightarrow}\left(V, D, v_{0}\right) \\
\downarrow p \quad \downarrow d r \\
\left(X, A, x_{0}\right) \stackrel{f}{\rightarrow}\left(Y, B, y_{0}\right) .
\end{gathered}
$$

3. Homotopy theories. A homotopy theory

$$
H=\{\pi, *, \partial\}
$$

consists of three functions $\pi, *$ and $\partial$ described as follows.

The first function $\pi$ associates with each triplet $\left(X, A, x_{0}\right)$ and each integer $m \geqq 0$ an abstrat set $\pi_{m}\left(X, A, x_{0}\right)$; we require that $\pi_{0}\left(X, A, x_{0}\right)$ be the set $P(X, A)$ of path-components of $X$ modulo $A$ and that $\pi_{m}\left(X, A, x_{0}\right)$ be a group in each of the following cases:

(HG1) $m \geqq 2$.

(HG2) $m=1$ and $A=x_{0}$.

(HG3) $m=1$ and $\left(X, A, x_{0}\right)$ is the associated triplet of some triplet of the form $\left(R, r_{0}, r_{0}\right)$.

Note. In the case (HG3), $\pi_{0}\left(A, x_{0}\right)$ is a group with usual multiplication among classes of loops.

The second function * associates with each continuous map $f:\left(X, A, x_{0}\right) \rightarrow\left(Y, B, y_{0}\right)$ and each integer $m \geqq 0$ an induced transformation

$$
f_{*}: \pi_{m}\left(X, A, x_{0}\right) \rightarrow \pi_{m}\left(Y, B, y_{0}\right) .
$$

We require that $f_{*}$ should be the natural induced transformation defined in $\S 2$ in case $m=0$ and that $f_{*}$ should be a homomorphism in each of the following cases:

(IT1) $m \geqq 2$. 
(IT2) $m=1, A=x_{0}$ and $B=y_{0}$.

(IT3) $m=1,\left(X, A, x_{0}\right)$ is the associated triplet of some triplet $\left(R, r_{0}, r_{0}\right),\left(Y, B, y_{0}\right)$ is the associated triplet of some triplet $\left(S, s_{0}, s_{0}\right)$, and $f$ is induced by a continuous map $\phi:\left(R, r_{0}\right) \rightarrow\left(S, s_{0}\right)$.

(IT4) $m=1, B=y_{0},\left(X, A, x_{0}\right)$ is the associated triplet of $\left(Y, B, y_{0}\right)$, and $f$ is the associated projection.

Note. In the case (IT3), $f_{*}: \pi_{0}\left(A, x_{0}\right) \rightarrow \pi_{0}\left(B, y_{0}\right)$ is also a homomorphism.

The third function $\partial$ associates with each triplet $\left(X, A, x_{0}\right)$ and each integer $m>0$ a boundary operation

$$
\partial: \pi_{m}\left(X, A, x_{0}\right) \rightarrow \pi_{m-1}\left(A, x_{0}\right) ;
$$

we require $\partial$ to be a homomorphism in each of the following cases:

(BO1) $m \geqq 2$.

(BO2) $m=1$ and $\left(X, A, x_{0}\right)$ is the associated triplet of some triplet $\left(R, r_{0}, r_{0}\right)$.

Furthermore, the collection $H=\{\pi, *, \partial\}$ must satisfy the following seven axioms.

Axiom I. If $f$ is the identity map on a triplet $\left(X, A, x_{0}\right)$, then $f_{*}$ is the identity on $\pi_{m}\left(X, A, x_{0}\right)$.

Axiom II. If $f:\left(X, A, x_{0}\right) \rightarrow\left(Y, B, y_{0}\right)$ and $g:\left(Y, B, y_{0}\right) \rightarrow\left(Z, C, z_{0}\right)$ are continuous maps, then $(g f)_{*}=g_{*} f_{*}$.

Axiom III. If the continuous maps $f:\left(X, A, x_{0}\right) \rightarrow\left(Y, B, y_{0}\right)$ and $g:\left(A, x_{0}\right) \rightarrow\left(B, y_{0}\right)$ are related by $g=f \mid\left(A, x_{0}\right)$, then $\partial f_{*}=g_{*} \partial$.

The foregoing three axioms are called the algebraic axioms.

Axiom IV (Homotopy Axiom). If the continuous maps $f, g$ : $\left(X, A, x_{0}\right) \rightarrow\left(Y, B, y_{0}\right)$ are homotopic, then $f_{*}=g_{*}$.

Let $\left(X, A, x_{0}\right)$ be any given triplet. Then the inclusion maps $i:\left(A, x_{0}\right) \rightarrow\left(X, x_{0}\right)$ and $j:\left(X, x_{0}\right) \rightarrow\left(X, A, x_{0}\right)$ together with the boundary operations $\partial$ give rise to a beginningless sequence

$$
\begin{gathered}
\ldots \stackrel{j_{*}}{\rightarrow} \pi_{m+1}\left(X, A, x_{0}\right) \stackrel{\partial}{\rightarrow} \pi_{m}\left(A, x_{0}\right) \stackrel{i_{*}}{\rightarrow} \pi_{m}\left(X, x_{0}\right) \stackrel{j_{*}}{\rightarrow} \pi_{m}\left(X, A, x_{0}\right) \stackrel{\partial}{\rightarrow} \cdots \\
\quad \ldots \stackrel{j_{*}}{\rightarrow} \pi_{1}\left(X, A, x_{0}\right) \stackrel{\partial}{\rightarrow} \pi_{0}\left(A, x_{0}\right) \stackrel{i_{*}}{\rightarrow} \pi_{0}\left(X, x_{0}\right) \stackrel{j_{*}}{\rightarrow} \pi_{0}\left(X, A, x_{0}\right)
\end{gathered}
$$

which will be called the homotopy sequence of the triplet $\left(X, A, x_{0}\right)$ in the homotopy theory $H$.

Axiom $\mathrm{V}$ (Exactness Axiom). The homotopy sequence of any triplet $\left(X, A, x_{0}\right)$ is weakly exact. 
This means that, if $\pi_{m}\left(X, x_{0}\right)=0$ for all $m \geqq 0$, then $\partial: \pi_{m}\left(X, A, x_{0}\right)$ $\approx \pi_{m-1}\left(A, x_{0}\right)$ for every $m>0$. Here, $S=0$ denotes that the set $S$ consists of a single element and $\tau: M \approx M$ denotes that the transformation $\tau$ maps $M$ onto $N$ in a one-to-one fashion.

Axiom VI (Fibering Axiom). If $\left(U, C, u_{0}\right)$ is the associated triplet of $\left(X, A, x_{0}\right)$ and if $p:\left(U, C, u_{0}\right) \rightarrow\left(X, A, x_{0}\right)$ is the associated projection, then

$$
p_{*}: \pi_{m}\left(U, C, u_{0}\right) \approx \pi_{m}\left(X, A, x_{0}\right), \quad m>0 .
$$

Axiom VII (Dimension Axiom). If $X=x_{0}$, then $\pi_{m}\left(X, x_{0}\right)=0$ for every $m \geqq 0$.

Let $H=\{\pi, *, \partial\}$ be a homotopy theory. The group $\pi_{m}\left(X, A, x_{0}\right)$, $m \geqq 2$, is called the $m$ th relative homotopy group of $X$ modulo $A$ at the basic point $x_{0}$ (in the homotopy theory $H$ ); and the group $\pi_{m}\left(X, x_{0}\right.$ ), $m \geqq 1$, is called the $m$ th (absolute) homotopy group of $X$ at the basic point $x_{0}$. In particular, $\pi_{1}\left(X, x_{0}\right)$ is usually called the fundamental group or the Poincare group of $X$ at the basic point $x_{0}$. For completeness, we shall also call $\pi_{m}\left(X, A, x_{0}\right)$ the $m$ th relative homotopy set of $X$ modulo $A$ at $x_{0}$ and $\pi_{m}\left(X, x_{0}\right)$ the $m$ th homotopy set of $X$ at $x_{0}$ for every $m \geqq 0$.

4. Properties. Most of the elementary results in homotopy theory can be deduced right from the axioms. Precisely, for any given homotopy theory

$$
H=\{\pi, *, \partial\},
$$

we shall give in the present section a few properties of $H$ as consequences of its definition given in $\S 3$.

(4.1) For every triplet $\left(X, A, x_{0}\right)$ and every $m \geqq 0, \pi_{m}\left(X, A, x_{0}\right)$ is nonempty and contains a special element 0 called the neutral element of $\pi_{m}\left(X, A, x_{0}\right)$.

In fact, 0 is the neutral element of $P(X, A)$ defined in $\$ 2$ if $m=0$. For $m>0,0$ is the image under $j_{*}$ of the group-theoretic neutral element of $\pi_{m}\left(X, x_{0}\right)$.

(4.2) If $f:\left(X, A, x_{0}\right) \rightarrow\left(Y, B, y_{0}\right)$ is a homotopy equivalence, then $f_{*}: \pi_{m}\left(X, A, x_{0}\right) \approx \pi_{m}\left(Y, B, y_{0}\right)$ for every $m \geqq 0$.

As immediate consequences of (4.2), we obtain the following three assertions:

(a) For every $m \geqq 0, \pi_{m}\left(X, A, x_{0}\right)$ is an invariant of the homotopy type of the triplet $\left(X, A, x_{0}\right)$.

(b) If $A$ is a deformation retract of $X$, then $i_{*}: \pi_{m}\left(A, x_{0}\right) \approx \pi_{m}\left(X, x_{0}\right)$ for every $m \geqq 0$. 
(c) If $X$ is contractible to the point $x_{0}$, then $\pi_{m}\left(X, x_{0}\right)=0$ for every $m \geqq 0$.

Now let $\left(X, A, x_{0}\right)$ be a given triplet. Consider the associated triplet $\left(U, C, u_{0}\right)$ of $\left(X, A, x_{0}\right)$ and the associated projection $p$ : $\left(U, C, u_{0}\right) \rightarrow\left(X, A, x_{0}\right)$. We have a diagram

$$
\pi_{m}\left(X, A, x_{0}\right) \stackrel{p_{*}}{\leftarrow} \pi_{m}\left(U, C, u_{0}\right) \stackrel{\partial}{\rightarrow} \pi_{m-1}\left(C, u_{0}\right)
$$

for every $m>0$. According to the fibering axiom, we have $p_{*}$ : $\pi_{m}\left(U, C, u_{0}\right) \approx \pi_{m}\left(X, A, x_{0}\right)$. Hence we obtain a natural transformation

$$
\nu=\partial \overrightarrow{p_{*}}: \pi_{m}\left(X, A, x_{0}\right) \rightarrow \pi_{m-1}\left(C, u_{0}\right)
$$

for every $m>0$. In the cases (HG1) through (HG3), $\nu$ is obviously a homomorphism.

$$
\nu: \pi_{m}\left(X, A, x_{0}\right) \approx \pi_{m-1}\left(C, u_{0}\right) \quad \text { for every } m>0 .
$$

The special case $A=x_{0}$ is of importance. In this case, $C$ becomes the space of loops in $X$ with basic point $x_{0}$ and $\nu$ is an isomorphism of $\pi_{m}\left(X, x_{0}\right)$ onto $\pi_{m-1}\left(C, u_{0}\right)$ for every $m>0$.

Next, let $\left(X, A, x_{0}\right)$ be a given triplet and $Y$ be any fiber space (in the sense of Serre) over a subspace $X_{0}$ of $X$ relative to a projection $f: Y \rightarrow X_{0}$. Let $B=f^{-1}(A)$ and pick a point $y_{0} \in f^{-1}\left(x_{0}\right)$. Thus we obtain a triplet $\left(Y, B, y_{0}\right)$ and a continuous map $f:\left(Y, B, y_{0}\right) \rightarrow\left(X, A, x_{0}\right)$. If $X_{0}$ contains the path-component of $X$ containing $x_{0}, f$ will be called a fibering map.

(4.4) The Fibering Theorem. If $f:\left(Y, B, y_{0}\right) \rightarrow\left(X, A, x_{0}\right)$ is a fibering map, then $f_{*}: \pi_{m}\left(Y, B, y_{0}\right) \approx \pi_{m}\left(X, A, x_{0}\right)$ for every $m>0$.

If $\left(U, C, u_{0}\right)$ denotes the associated triplet of $\left(X, A, x_{0}\right)$, then it is well-known that $U$ is a fiber space over the path-component of $X$ containing $x_{0}$ and hence the associated projection $p:\left(U, C, u_{0}\right) \rightarrow\left(X, A, x_{0}\right)$ is a fibering map. Therefore, (4.4) is a generalization of the Fibering Axiom.

Let $X_{0}$ denote the path-component of $X$ containing $x_{0}$ and $A_{0}$ $=A \cap X_{0}$, then a direct consequence of (4.4) is that the inclusion map $i$ induces $i_{*}: \pi_{m}\left(X_{0}, A_{0}, x_{0}\right) \approx \pi_{m}\left(X, A, x_{0}\right)$ for every $m>0$.

By a triple $(X, A, B)$, we mean a topological space $X$ together with two nonvoid subspaces $A$ and $B$ such that $A \supset B$. Pick a basic point $x_{0}$ in $B$. The inclusion maps

$$
i:\left(A, B, x_{0}\right) \subset\left(X, B, x_{0}\right), \quad j:\left(X, B, x_{0}\right) \subset\left(X, A, x_{0}\right)
$$

give rise to the induced transformations $i_{*}$ and $j_{*}$. On the other hand, 
the inclusion map $k:\left(A, x_{0}\right) \subset\left(A, B, x_{0}\right)$ has the induced transformations $k_{*}$. Define a boundary operation

$$
\bar{\partial}: \pi_{m}\left(X, A, x_{0}\right) \rightarrow \pi_{m-1}\left(A, B, x_{0}\right)
$$

for every $m>0$ by taking the composition $\bar{\partial}=k_{*} \partial$ of

$$
\pi_{m}\left(X, A, x_{0}\right) \stackrel{\partial}{\rightarrow} \pi_{m-1}\left(A, x_{0}\right) \stackrel{k_{*}}{\rightarrow} \pi_{m-1}\left(A, B, x_{0}\right) .
$$

Further, let 0 denote a trivial set consisting of a single element called its neutral element and let $\bar{\partial}: \pi_{0}\left(X, A, x_{0}\right) \rightarrow 0$ denote the trivial transformation. Thus we obtain a beginningless sequence

$$
\begin{gathered}
\ldots \stackrel{\bar{j}_{*}}{\rightarrow} \pi_{m+1}\left(X, A, x_{0}\right) \stackrel{\bar{\partial}}{\rightarrow} \pi_{m}\left(A, B, x_{0}\right) \stackrel{\bar{i}_{*}}{\rightarrow} \pi_{m}\left(X, B, x_{0}\right) \stackrel{\bar{j}_{*}}{\rightarrow} \pi_{m}\left(X, A, x_{0}\right) \stackrel{\bar{\partial}}{\rightarrow} \cdots \\
\ldots \stackrel{\bar{\partial}}{\rightarrow} \pi_{0}\left(A, B, x_{0}\right) \stackrel{\bar{i}_{*}}{\rightarrow} \pi_{0}\left(X, B, x_{0}\right) \stackrel{\bar{j}_{*}}{\rightarrow} \pi_{0}\left(X, A, x_{0}\right) \stackrel{\bar{\partial}}{\rightarrow} 0
\end{gathered}
$$

called the homotopy sequence of the triple $(X, A, B)$ at the basic point $x_{0}$. If $B=x_{0}$, then this reduces to the homotopy sequence of the triplet $\left(X, A, x_{0}\right)$ augmented on its right end by a trivial set 0 . In this sequence, every set has a specific neutral element. By the kernel of a transformation in this sequence, we mean the inverse image of the neutral element.

(4.5) The Exactness Theorem. The homotopy sequence of any triple $(X, A, B)$ at any basic point $x_{0} \in B$ is exact; that is to say, the kernel of every transformation in the sequence coincides with the image of the preceding transformation.

Note. (4.5) strengthens as well as generalizes the Exactness Axiom.

(4.6) The Commutativity Theorem. $\pi_{m}\left(X, A, x_{0}\right)$ is an abelian group in each of the following cases:

(C1) $m=1, X$ is an $H$-space [11, p. 474], with $x_{0}$ as a two-sided homotopy unit, and $A=x_{0}$.

(C2) $m=2$ and $\left(X, A, x_{0}\right)$ is the associated triplet of a triplet of the form $\left(R, r_{0}, r_{0}\right)$.

(C3) $m=2$ and $A=x_{0}$.

(C4) $m>2$.

REMARK. If $n \geqq 0$ is a given integer and if $H=\{\pi, *, \partial\}$ is defined only for the dimensions $m \leqq n$ such that the axioms of $\S 3$ are satisfied for these dimensions, then the assertions in this section are also true for these dimensions. In fact, only these dimensions are used in the proofs. 
5. Existence. The purpose of this section is to prove the existence of a homotopy theory. In fact, we shall construct a homotopy theory $H=\{\pi, *, \partial\}$ by induction as follows.

According to the definition, $\pi_{0}\left(X, A, x_{0}\right)$ and $f_{*}: \pi_{0}\left(X, A, x_{0}\right)$ $\rightarrow \pi_{0}\left(Y, B, y_{0}\right)$ are well-defined for every triplet $\left(X, A, x_{0}\right)$ and every continuous map $f:\left(X, A, x_{0}\right) \rightarrow\left(Y, B, y_{0}\right)$.

Let $n \geqq 1$ be a given integer and assume that we have already constructed the homotopy sets $\pi_{m}\left(X, A, x_{0}\right)$, for each $m<n$ and triplet $\left(X, A, x_{0}\right)$, together with the induced transformations $f_{*}$ and the boundary operations $\partial$ on these homotopy sets, such that all conditions and axioms are satisfied.

First, let us construct the homotopy set $\pi_{n}\left(X, A, x_{0}\right)$ of a given triplet $\left(X, A, x_{0}\right)$. Consider the associated triplet $\left(U, C, u_{0}\right)$ of $\left(X, A, x_{0}\right)$ and the associated projection $p:\left(U, C, u_{0}\right) \rightarrow\left(X, A, x_{0}\right)$. We define

$$
\pi_{n}\left(X, A, x_{0}\right)=\pi_{n-1}\left(C, u_{0}\right) .
$$

Next, let us define the boundary operation $\partial: \pi_{n}\left(X, A, x_{0}\right)$ $\rightarrow \pi_{n-1}\left(A, x_{0}\right)$. Let $q=p \mid\left(C, u_{0}\right)$. Then we define

$$
\partial=q_{*}: \pi_{n-1}\left(C, u_{0}\right) \rightarrow \pi_{n-1}\left(A, x_{0}\right) .
$$

Finally, let $f:\left(X, A, x_{0}\right) \rightarrow\left(Y, B, y_{0}\right)$ be a given continuous map. We shall construct the induced transformation $f_{*}: \pi_{n}\left(X, A, x_{0}\right)$ $\rightarrow \pi_{n}\left(Y, B, y_{0}\right)$ as follows. Let $\left(V, D, v_{0}\right)$ denote the associated triplet of $\left(Y, B, y_{0}\right)$ with associated projection $r:\left(V, D, v_{0}\right) \rightarrow\left(Y, B, y_{0}\right)$. According to $\S 2, f$ induces a continuous map $\phi:\left(U, C, u_{0}\right) \rightarrow\left(V, D, v_{0}\right)$. Let $\psi=\phi \mid\left(C, u_{0}\right)$. Then we define

$$
f_{*}=\psi_{*}: \pi_{n-1}\left(C, u_{0}\right) \rightarrow \pi_{n-1}\left(D, v_{0}\right) .
$$

It is verified that all the conditions and axioms in $\$ 3$ are satisfied. This completes the inductive construction of a homotopy theory $H$ $=\{\pi, *, \partial\}$. This theory $H$ will be called the natural homotopy theory.

6. Uniqueness. Two homotopy theories $H=\{\pi, *, \partial\}$ and $H^{\prime}$ $=\left\{\pi^{\prime}, \#, \partial^{\prime}\right\}$ are said to be equivalent if there exists, for each triplet $\left(X, A, x_{0}\right)$ and each $m \geqq 0$, a transformation

$$
h_{m}: \pi_{m}\left(X, A, x_{0}\right) \rightarrow \pi_{m}^{\prime}\left(X, A, x_{0}\right)
$$

satisfying the conditions:

(E1) $h_{0}$ is the identity on $\pi_{0}\left(X, A, x_{0}\right)=\pi_{0}^{\prime}\left(X, A, x_{0}\right)$.

(E2) $h_{m}$ is a homomorphism if $m \geqq 2$ or if $m=1$ and $A=x_{0}$.

(E3) $h_{m}: \pi_{m}\left(X, A, x_{0}\right) \approx \pi_{m}^{\prime}\left(X, A, x_{0}\right)$.

(E4) $h_{m} f_{*}=f \sharp h_{m}$. 
(E5) $h_{m-1} \partial=\partial^{\prime} h_{m}$

A collection of transformations $h=\left\{h_{m}\right\}$ satisfying the conditions (E1) through (E5) is called an equivalence between $H$ and $H^{\prime}$ and is denoted by $h: H \approx H^{\prime}$.

We are going to prove the uniqueness theorem that any two homotopy theories $H$ and $H^{\prime}$ are equivalent. For this purpose, we shall construct an equivalence $h: H \approx H^{\prime}$ as follows.

The transformation $h_{0}$ is defined by (E1). Let $n \geqq 1$ be a given integer and assume that we have already constructed the transformations $h_{m}$ for each $m<n$ and each triplet $\left(X, A, x_{0}\right)$ such that (E1)-(E5) are satisfied. Let us construct $h_{n}$ as follows.

Let $\left(X, A, x_{0}\right)$ be any triplet. Consider the associated triplet $\left(U, C, u_{0}\right)$ of $\left(X, A, x_{0}\right)$ and the associated projection $p:\left(U, C, u_{0}\right)$ $\rightarrow\left(X, A, x_{0}\right)$. According to the Fibering Axiom, we have

$$
p_{*}: \pi_{n}\left(U, C, u_{0}\right) \approx \pi_{n}\left(X, A, x_{0}\right), \quad p_{\sharp}: \pi_{n}^{\prime}\left(U, C, u_{0}\right) \rightarrow \pi_{n}^{\prime}\left(X, A, x_{0}\right) .
$$

It is well-known that $U$ is contractible to the point $u_{0}$; hence $\pi_{m}\left(U, u_{0}\right)=0$ for every $m \geqq 0$ by the Axioms I, II, IV and VII. According to the Exactness Axiom, this implies

$$
\partial: \pi_{n}\left(U, C, u_{0}\right) \approx \pi_{n-1}\left(C, u_{0}\right), \partial^{\prime}: \pi_{n}^{\prime}\left(U, C, u_{0}\right) \approx \pi_{n-1}^{\prime}\left(C, u_{0}\right) .
$$

By our assumption of induction, we have

$$
h_{n-1}: \pi_{n-1}\left(C, u_{0}\right) \approx \pi_{n-1}^{\prime}\left(C, u_{0}\right) .
$$

Hence we may define a transformation

$$
h_{n}: \pi_{n}\left(X, A, x_{0}\right) \rightarrow \pi_{n}^{\prime}\left(X, A, x_{0}\right)
$$

by taking the composition

$$
h_{n}=p * \partial^{\prime-1} h_{n-1} \partial p_{*}^{-1} \text {. }
$$

It can be verified that $h_{n}$ satisfies the conditions (E1) through (E5). This completes the inductive construction of $h=\left\{h_{m}\right\}$ and proves the uniqueness theorem.

The uniqueness theorem shows that the natural homotopy theory constructed in $\$ 5$ is essentially the only homotopy theory.

The equivalence $h=\left\{h_{m}\right\}$ constructed above will be called the natural equivalence between the homotopy theories $H$ and $H^{\prime}$.

7. The uniqueness of equivalence. The purpose of this section is to show that the natural equivalence constructed in $\$ 6$ is the only equivalence ${ }^{3}$ between any two given homotopy theories $H=\{\pi, *, \partial\}$

3 As a remark given by A. D. Wallace at the meeting, this means that there is a unique way to prove the uniqueness theorem. 
and $H^{\prime}=\left\{\pi^{\prime}, \#, \partial^{\prime}\right\}$.

By an admissible transformation

$$
k=\left\{k_{m}\right\}: H \rightarrow H^{\prime}
$$

of $H$ into $H^{\prime}$, we mean for each triplet $\left(X, A, x_{0}\right)$ and each integer $m \geqq 0$ a transformation

$$
k_{m}: \pi_{m}\left(X, A, x_{0}\right) \rightarrow \pi_{m}^{\prime}\left(X, A, x_{0}\right)
$$

satisfying the conditions:

(AT1) $k_{0}$ is the identity.

(AT2) $k_{m} f_{*}=f_{*} k_{m}$.

(AT3) $k_{m-1} \partial=\partial^{\prime} k_{m}$.

By (E1), (E4) and (E5), every equivalence between $H$ and $H^{\prime}$ is an admissible transformation. Conversely, we shall prove that every admissible transformation $k$ coincides with the natural equivalence $h$, i.e., $k_{m}=h_{m}$ for every $m \geqq 0$.

By definition, we have $k_{0}=h_{0}$. We shall prove the assertion by induction. Let $n>0$ be a given integer and assume that $k_{m}=h_{m}$ for every $m<n$. We are going to show that $k_{n}=h_{n}$.

Let $\left(X, A, x_{0}\right)$ be any triplet. Consider the associated triplet $\left(U, C, u_{0}\right)$ of $\left(X, A, x_{0}\right)$ and the associated projection $p:\left(U, C, u_{0}\right)$ $\rightarrow\left(X, A, x_{0}\right)$. In the following diagram

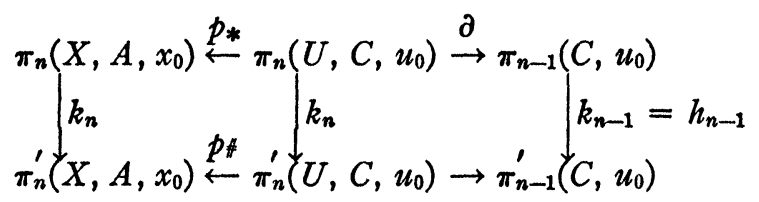

we have commutativity in both rectangles as required by (AT2) and (AT3). Hence we obtain

$$
k_{n}=p * k_{n} p_{*}^{-1}=p * \partial^{\prime-1} h_{n-1} \partial p_{*}^{-1}=h_{n} .
$$

This result implies that the natural equivalence $h$ is the only equivalence between $H$ and $H^{\prime}$. Furthermore, in order to construct geometrically the natural equivalence between two homotopy theories given by geometric definitions, it suffices to establish an admissible transformation by means of some natural geometric method.

8. The role of the basic point. In this section, we shall continue our axiomatic approach and study the role played by the basic point in a homotopy theory $H=\{\pi, *, \partial\}$.

Let us consider a given space $X$ and two given points $x_{0}, x_{1}$ connected by a path 


$$
\sigma: I \rightarrow X, \quad \sigma(0)=x_{0}, \quad \sigma(1)=x_{1} .
$$

By definition, we have $\pi_{0}\left(X, x_{0}\right)=P(X)=\pi_{0}\left(X, x_{1}\right)$. Further, since $x_{0}$ and $x_{1}$ are contained in the same path-component of $X$, the neutral element of $\pi_{0}\left(X, x_{0}\right)$ is the same as that of $\pi_{0}\left(X, x_{1}\right)$. For the relations between the homotopy groups $\pi_{m}\left(X, x_{0}\right)$ and $\pi_{m}\left(X, x_{1}\right), m \geqq 1$, let us consider the spaces of loops $W_{0}, W_{1}$ in $X$ with basic points at $x_{0}, x_{1}$ respectively. Let $w_{0} \in W_{0}$ and $w_{1} \in W_{1}$ denote the degenerate loops.

The path $\sigma$ induces a continuous map $\xi: W_{1} \rightarrow W_{0}$ defined as follows: for each $w \in W_{1}, \xi(w) \in W_{0}$ is the loop defined by

$$
[\xi(w)](t)=\left\{\begin{array}{lr}
\sigma(3 t), & \text { if } 0 \leqq t \leqq 1 / 3, \\
w(3 t-1), & \text { if } 1 / 3 \leqq t \leqq 2 / 3, \\
\sigma(3-3 t) & \text { if } 2 / 3 \leqq t \leqq 1 .
\end{array}\right.
$$

On the other hand, $\sigma$ also induces a path $\eta: I \rightarrow W_{0}$ defined as follows: for each real number $s \in I, \eta(s) \in W_{0}$ is the loop defined by

$$
[\eta(s)](t)=\left\{\begin{array}{lr}
\sigma(3 s t), & \text { if } 0 \leqq t \leqq 1 / 3, \\
\sigma(s), & \text { if } 1 / 3 \leqq t \leqq 2 / 3, \\
\sigma(3 s-3 s t), & \text { if } 2 / 3 \leqq t \leqq 1 .
\end{array}\right.
$$

Then, obviously we have $\eta(0)=w_{0}$ and $\eta(1)=\xi\left(w_{1}\right)$.

By a system of operations in a homotopy theory $H=\{\pi, *, \partial\}$, we mean for each path $\sigma: I \rightarrow X$ in any topological space $X$ and each integer $m \geqq 0$ a transformation

$$
\sigma_{m}: \pi_{m}\left(X, x_{1}\right) \rightarrow \pi_{m}\left(X, x_{0}\right), \quad x_{0}=\sigma(0), \quad x_{1}=\sigma(1)
$$

satisfying the conditions:

(SO1) $\sigma_{0}$ is the identity on $\pi_{0}\left(X, x_{1}\right)=\pi_{0}\left(X, x_{0}\right)$.

(SO2) If $m>0$ and if $\xi:\left(W_{1}, w_{1}\right) \rightarrow\left(W_{0}, \xi\left(w_{1}\right)\right)$ and $\eta: I \rightarrow W_{0}$ are induced by $\sigma$, then commutativity holds in the following diagram

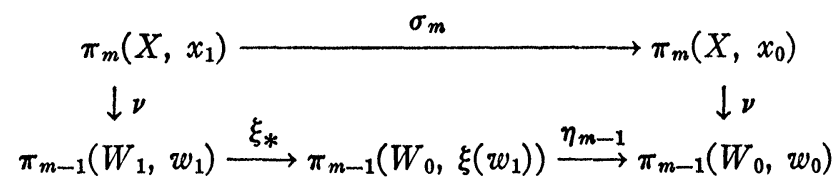

where $\nu$ denotes the natural transformation.

According to (4.3), $\nu^{-1}$ is well-defined. Hence we obtain

$$
\sigma_{m}=\nu^{-1} \eta_{m-1} \xi_{*} \nu
$$

It is easy to see that $\sigma_{m}$ is a homomorphism if $m>0$. By (SO1) and (8.1), the inductive proof of the following theorem is obvious. 
(8.2) In any given homotopy theory $H=\{\pi, *, \partial\}$, there exists one and only one system of operations. Furthermore, for any two given homotopy theories $H$ and $H^{\prime}$, the natural equivalence $h: H \approx H^{\prime}$ commutes with the operations in $H$ and $H^{\prime}$.

The usual general properties of the system of operations in $H$ can be deduced from (SO1) and (SO2) without using the traditional geometric meaning of these operations. Hence one may prove the following assertion.

(8.3) For any given topological space $X$ and any given integer $m \geqq 1$, the collection of the homotopy groups $\left\{\pi_{m}\left(X, x_{0}\right) \mid x_{0} \in X\right\}$ in a homotopy theory $H=\{\pi, *, \partial\}$ forms a local system of groups in $X$ in the sense of Steenrod.

Consequently, if $\sigma: I \rightarrow X$ is a path joining $x_{0}$ to $x_{1}$, then $\sigma_{m}: \pi_{m}\left(X, x_{1}\right)$ $\approx \pi_{m}\left(X, x_{0}\right)$. If $X$ is pathwise connected, then all the groups $\pi_{m}\left(X, x_{0}\right)$, $x_{0} \in X$, are isomorphic and the abstract group $\pi_{m}(X)$ which is isomorphic to $\pi_{m}\left(X, x_{0}\right)$ for each $x_{0} \in X$ will be called the $m$ th (abstract) homotopy group of $X$.

Another consequence of (8.3) is that for each $m \geqq 1$, the fundamental group $\pi_{1}\left(X, x_{0}\right)$ acts on the left of $\pi_{m}\left(X, x_{0}\right)$ as a group of operators. In the special case $m=1$, one can easily see that, for any two elements $g$ and $h$ in $\pi_{1}\left(X, x_{0}\right), h$ acts on $g$ as follows:

$$
h(g)=h g h^{-1} \text {. }
$$

Next, let us construct the operations in the relative homotopy sets. Consider a given topological space $X$, a given subspace $A$ of $X$, and two given points $x_{0}, x_{1}$ connected by a path $\sigma: I \rightarrow A$ with $\sigma(0)=x_{0}$ and $\sigma(1)=x_{1}$.

Let $\left(U_{0}, C_{0}, u_{0}\right)$ and $\left(U_{1}, C_{1}, u_{1}\right)$ denote the associated triplets of ( $\left.X, A, x_{0}\right)$ and $\left(X, A, x_{1}\right)$ respectively. The path $\sigma$ induces a continuous map $\xi: C_{1} \rightarrow C_{0}$ defined as follows: for each $u \in C_{1}, \xi(u) \in C_{0}$ is the path defined by

$$
[\xi(u)](t)= \begin{cases}\sigma(2 t), & \text { if } 0 \leqq t \leqq 1 / 2, \\ u(2 t-1), & \text { if } 1 / 2 \leqq t \leqq 1 .\end{cases}
$$

On the other hand, $\sigma$ also induces a path $\eta: I \rightarrow C_{0}$ defined as follows: for each $s \in I, \eta(s) \in C_{0}$ is the path defined by

$$
[\eta(s)](t)= \begin{cases}\sigma(2 s t), & \text { if } 0 \leqq t \leqq 1 / 2, \\ \sigma(s), & \text { if } 1 / 2 \leqq t \leqq 1\end{cases}
$$

Obviously we have $\eta(0)=u_{0}$ and $\eta(1)=\xi\left(u_{1}\right)$.

For each $m \geqq 0$, we define an operation 


$$
\sigma_{m}: \pi_{m}\left(X, A, x_{1}\right) \rightarrow \pi_{m}\left(X, A, x_{0}\right)
$$

as follows: $\sigma_{0}$ is the identity on $\pi_{0}\left(X, A, x_{1}\right)=\pi_{0}\left(X, A, x_{0}\right)$; if $m>0$, then $\sigma_{m}$ is uniquely defined by the commutativity of the following diagram

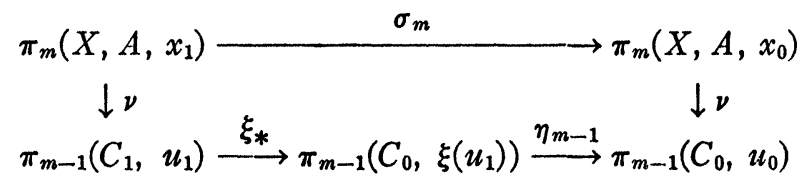

where $\nu$ denotes the natural transformations. Hence,

$$
\sigma_{m}=\nu^{-1} \eta_{m-1} \xi_{*} \nu
$$

One can easily establish the usual general properties of these operations. In particular, $\sigma_{m}: \pi_{m}\left(X, A, x_{1}\right) \approx \pi_{m}\left(X, A, x_{0}\right)$ and, if $m \geqq 2$, the relative homotopy groups $\left\{\pi_{m}\left(X, A, x_{0}\right) \mid x_{0} \in A\right\}$ form a local system of groups in $A$. Consequently, $\pi_{1}\left(A, x_{0}\right)$ acts on the left of $\pi_{m}\left(X, A, x_{0}\right)$ as a group of operators for every $m \geqq 2$.

If $A$ is pathwise connected, then, for a given $m \geqq 2$, all the groups $\pi_{m}\left(X, A, x_{0}\right), x_{0} \in A$, are isomorphic. The abstract group $\pi_{m}(X, A)$ which is isomorphic to $\pi_{m}\left(X, A, x_{0}\right)$ for each $x_{0} \in A$ will be called the $m$ th (abstract) relative homotopy group of $X$ modulo $A$.

9. Remarks and problems. Although a homotopy theory looks quite like a homology theory, they differ in the following aspects:

(1) The excision axiom holds in homology theory but not in homotopy theory; on the other hand, the fibering axiom holds in homotopy theory but not in homology theory.

(2) The uniqueness of homotopy theory is proved for all possible triplets, while that of homology theory is proved only for triangulable pairs. For general topological spaces, there are essentially different homology theories.

(3) In the homotopy sets $\pi_{m}\left(X, A, x_{0}\right)$, the basic point $x_{0}$ plays an important role, while the homology groups $H_{m}(X, A)$ do not depend on any basic point.

(4) All the homology groups $H_{m}(X, A), m \geqq 0$, are abelian groups. On the other hand, $\pi_{2}\left(X, A, x_{0}\right)$ is in general a nonabelian group, and $\pi_{0}\left(X, A, x_{0}\right), \pi_{1}\left(X, A, x_{0}\right)$ are in general not groups.

Hence, it is natural to expect that the homotopy groups are in general different from the corresponding homology groups. This was known to Poincare for the fundamental groups. The first higher dimensional example was given by Hopf [4], namely, $\pi_{3}\left(S^{2}\right) \approx Z$ while 
$H_{3}\left(S^{2}\right)=0$. Since then, numerous examples have been given in the literature.

Since the excision axiom does not hold in the homotopy theory, it is desirable to invent something which would measure the extent by which the excision axiom fails. This was solved by Blakers and Massey [1] by introducing their homotopy groups of triads. These groups as well as Freudenthal's suspension [3], can be conveniently defined in terms of spaces of paths [6], and hence can be fixed nicely into the present axiomatic scheme.

However, there are important operations in homotopy theory, such as the Whitehead products [15], which are defined by means of some specific geometrical constructions. So far, the author fails to present new definitions of these operations so that they might be fixed into the axiomatic scheme without appeal to the geometric representation of the homotopy groups.

Originally the question of axiomatizing the homotopy groups was considered important because it was hoped that such an axiomatization would lead to important new results or would simplify proofs of existing theorems. The proofs of the elementary properties of $\$ 4$ and many others are improved by this axiomatization. However, it is not likely that the axiomatization will help to simplify the proofs of such highly geometric theorems as the homotopy addition theorem, etc., because these theorems are essentially of the geometric representations of the homotopy groups.

\section{BIBLIOGRAPHY}

1. A. L. Blakers and W. S. Massey, The homotopy groups of a triad. I, Ann. of Math. vol. 53 (1951) pp. 161-205.

2. S. Eilenberg and N. E. Steenrod, Axiomatic approach to homology theory, Proc. Nat. Acad. Sci. U.S.A. vol. 31 (1945) pp. 117-120.

3. H. Freudenthal, Über die Klassen der Sphärenabbildungen. I. Grosse Dimension, Compositio Math. vol. 5 (1938) pp. 299-314.

4. H. Hopf, Über die Abbildungen der dreidimensionalen Sphäre auf die Kugelfläche, Math. Ann. vol. 104 (1931) pp. 637-665.

5. S. T. Hu, An exposition of the relative homotopy theory, Duke Math. J. vol. 14 (1947) pp. 991-1033.

6. - Homotopy properties of the space of continuous paths. II. The general case with arbitrary boundary sets, Portugaliae Mathematica vol. 11 (1952) pp. 41-50.

7. W. Hurewicz, Beiträge zur Topologie der Deformationen. I-IV, Koninklijke Nederlandsche Akademie van Wetenschappen, Proceedings vol. 38 (1935) pp. 112-119, 521-528, vol. 39 (1936) pp. 117-126, 215-224.

8. W. Hurewicz and N. E. Steenrod, Homotopy relations in fiber spaces, Proc. Nat. Acad. Sci. U.S.A. vol. 27 (1941) pp. 60-64.

9. H. Poincaré, Analysis situs, J. École Polytech. (2) vol. 1 (1892) pp. 1-121. 
10. - $5^{\circ}$ Complement d l'analysis situs, Rend. Circ. Mat. Palermo vol. 18 (1904) pp. 45-110.

11. J.-P. Serre, Homologie singulière des espaces fibres, Ann. of Math. (2) vol. 54 (1951) pp. 425-505.

12. E. H. Spanier, Borsuk's cohomotopy groups, Ann. of Math. (2) vol. 50 (1949) pp. 203-245.

13. N. E. Steenrod, Topology of fibre bundles, Princeton Math. Series, no. 14, 1951.

14. H. Tietze, Über die topologischen Invarianten mehrdimensionalen Mannigfaltigkeiten, Monatshefte für Mathematik und Physik vol. 19 (1908) pp. 1-118.

15. J. H. C. Whitehead, On adding relations to homotopy groups, Ann. of Math. (2) vol. 42 (1941) pp. 409-428.

16. - On the groups $\pi_{r}\left(V_{n, m}\right)$ and sphere bundles, Proc. London Math. Soc. (2) vol. 48 (1944) pp. 243-291.

17. J. Milnor, Construction of universal bundles, I, Ann. of Math. vol. 63 (1956) pp. 272-284 (added in proof).

UnIVERSITY OF GeORGIA 